\title{
ANÁLISIS
}

\section{EL PAPEL Y SUS POSIBILIDADES MULTIMEDIA EN LA}

BIBLIOTECA

\section{Fernando Juárez-Urquijo}

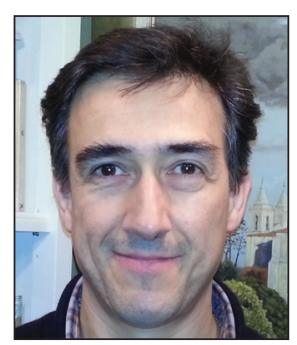

Fernando Juárez-Urquijo es bibliotecario y responsable web del Ayuntamiento de Muskiz (Bizkaia). Experimenta con la implementación de tecnologías web para imaginar servicios bibliotecarios de base. Ha publicado varios artículos y comunicaciones e impartido docencia especializada para profesionales sobre la web social y la web móvil aplicadas a las bibliotecas. Es miembro del Grupo ThinkEPI, representante (a propuesta de Aldee) del sector de archivos, bibliotecas y centros de documentación en el Consejo Vasco de Cultura y miembro del grupo de validación del Plan Vasco de Lectura. Mantiene junto a Natalia Arroyo-Vázquez y José-Antonio Merlo la 3á edición de Biblioblog.

http://orcid.org/0000-0002-8466-4511

Muskizko Udal Liburutegia Cendeja, 29. 48550 Muskiz, Bizkaia, España ferjur@gmail.com

\section{Resumen}

Internet, los nuevos dispositivos móviles y el almacenamiento en la nube están cambiando los hábitos de consumo de información. Se necesitan capacidad de almacenamiento y dispositivos lectores pero también se valoran la ubicuidad y la sincronización de datos entre diferentes aparatos. Se prima el acceso frente al almacenamiento. La web como servicio está acelerando la obsolescencia de soportes digitales que apenas cuentan con 20 años de existencia y potencia las opciones multimedia del papel, un soporte tradicional que facilita la relación persona/dispositivo móvil. Se exponen algunas experiencias realizadas en la Biblioteca de Muskiz explorando las nuevas oportunidades multimedia del papel.

\section{Palabras clave}

Soporte papel, Cloud computing, Biblioteca, Códigos bidimensionales, Realidad aumentada, Biblioteca de Muskiz.

\section{Title: Paper and its multimedia possibilities in the library}

\section{Abstract}

The Internet, the new mobile devices and cloud storage are changing the way in which people deal with information; we still need great storage capacity and information readers but we also value ubiquity and data synchronization between the different devices that we are using. Access has become more important than storage. The web as a tool is leading to the obsolescence of digital media that are just 20 years old and boosts the multimedia possibilities of paper, an analog medium that facilitates the relationship between people and mobile devices. We go through some of the experiences carried out in the Muskiz public library exploring the new multimedia possibilities of paper.

\section{Keywords}

Hard copy, Cloud computing, Library, Two-dimensional codes, Augmented reality, Muskiz library.

Juárez-Urquijo, Fernando (2013). "El papel y sus posibilidades multimedia en la biblioteca". El profesional de la información, enero-febrero, v. 22, n. 1, pp. 62-67.

http://dx.doi.org/10.3145/epi.2013.ene.08

\section{El papel en la época del cloud computing}

El papel es un soporte que parecía destinado a la marginación en el nuevo entorno digital caracterizado por el cloud computing (aplicaciones instaladas en servidores ubicados en lugares remotos de internet -y paraderos desconocidos para el usuario- pero accesibles desde cualquier sitio). Sin embargo, y curiosamente, hoy vuelve a adquirir un importante protagonismo gracias a los códigos bidimensionales (como los QRs) y a la realidad aumentada.

La Web está cambiando los hábitos y la forma de llegar a la información, y también la forma en que las bibliotecas ofrecen sus servicios, cada vez más accesibles desde cualquier 
lugar (Arroyo-Vázquez, 2009). Con el almacenamiento en la nube, la movilidad de los usuarios, los nuevos dispositivos móviles, el geoposicionamiento y la posibilidad de combinar contenido virtual con el entorno circundante, el papel adquiere nuevas connotaciones. Sin perder su naturale$\mathrm{za}^{1}$ se revela como soporte óptimo para el acceso a innovadoras formas de información multimedia.

En la nueva relación persona/dispositivo que propicia la nube, el papel puede facilitar el acceso a contenidos multimedia y activar procesos que facilitan la combinación de elementos reales con objetos virtuales (realidad aumentada).

\section{Facilitador de la relación persona/dispositivo}

El dispositivo móvil tiene muchas ventajas (información accesible desde cualquier lugar y almacenada en nuestro bolsillo) pero también, siendo sinceros, sus desventajas. El acceso a contenidos desde los terminales móviles vía teclado no es muy cómodo; movilidad, tránsito y falta de tiempo suelen ir en el mismo lote y todo aquello que evite teclear es un gran avance.

Aunque en principio el hipertexto y el formato papel no tienen una buena relación ${ }^{2}$, la nube y los códigos bidimensionales permiten revertir esta situación, ya que pueden codificar varios miles de caracteres ${ }^{3}$, son resistentes a posibles daños gracias a las fórmulas de corrección de errores que utilizan, se amoldan a todo tipo de superficies y encuentran en el papel un buen soporte por su portabilidad, resistencia y bajo coste. Habilitan la interacción propia de internet en un soporte no digital.

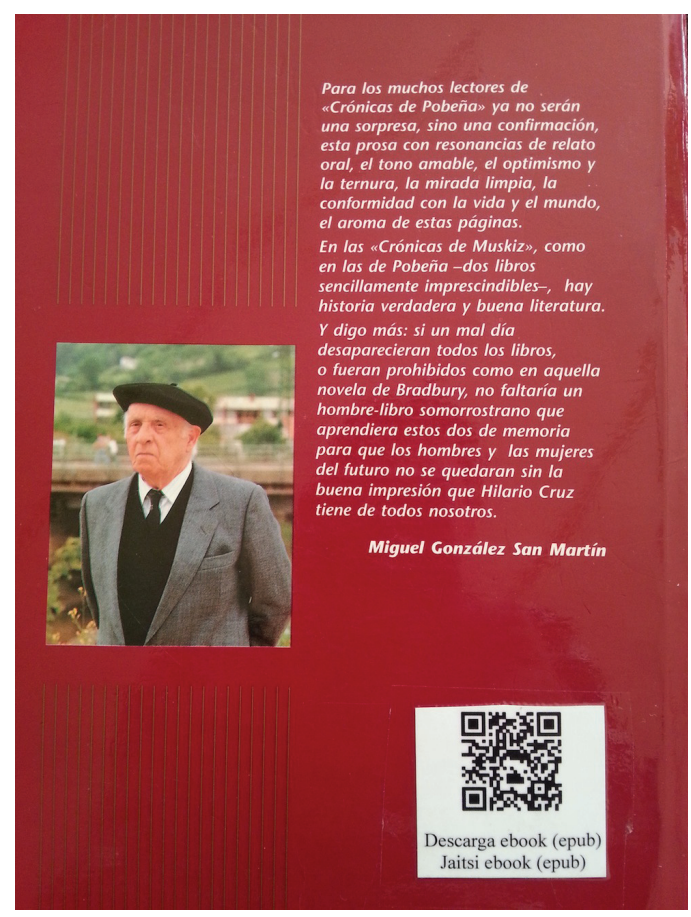

Figura 1. Detalle de la cubierta de Crónicas de Muskiz con el QR
Entre los numerosos tipos de códigos bidimensionales existentes, el QR (quick response code) goza de gran aceptación. Su estándar internacional ISO/IEC18004 fue aprobado en junio de 2000 y existen muchas soluciones para su generación y uso con coste cero. La inclusión de software que lee códigos QR en los smartphones ha facilitado nuevos usos orientados al consumidor como el dejar de introducir de forma manual los contactos y los urls.

El QR sobre papel puede contener información fija o actualizable. Si es fija, lo que se ve al escanear el código es el objeto correspondiente (un texto, los datos de una tarjeta de visita, un número de teléfono, una dirección postal, un mensaje informativo, una referencia bibliográfica, un mapa...). Si es actualizable lo habitual es que nos dirija a un url con contenido qué lógicamente puede ser modificado o tenerlo siempre actualizado. Este es un aspecto muy importante porque cambia la naturaleza del soporte papel al permitir modificar la información que contiene. Por ejemplo, si una biblioteca realiza un marcapáginas en papel con un calendario de sus actividades, aparecerán las del momento en el que se imprimió, pero el mismo marcapáginas con un QR que redireccione al apartado "Agenda" de una web, hace que indirectamente el soporte papel permanezca vigente y actualizado.

Los códigos bidimensionales permiten disponer de la interacción propia de internet en un soporte no digital

\section{Posibilidades de uso en la biblioteca. El caso de Mus- kiz}

La sencillez del mecanismo de creación y uso, su estética reconocible y el efecto de curiosidad que provocan, convierten los QRs en un potente instrumento para la difusión de servicios bibliotecarios. Se señalan entre otras sus posibilidades para mejorar la difusión de guías de recursos, descarga de contenidos o acceso a versión electrónica de artículos (Leiva, 2012). Uno de los primeros estudios sobre posibles usos del QR en la biblioteca se realizó en la University of Huddersfield, Reino Unido (Walsh, 2010), y en España, en la Universidad Politécnica de Valencia (Abarca et al., 2011).

En la Biblioteca municipal de Muskiz ${ }^{4}$ se emplean los QRs sobre papel para:

\section{- Mejorar la preservación y el acceso a la colección local}

La biblioteca posee un único ejemplar de los dos libros que publicó Hilario Cruz, un autor local ya fallecido. Se ha realizado una edición electrónica de ambos en formato epub y se facilita la descarga con un QR colocado en la cubierta de los libros. 


\section{- Complementar artículos y comunicaciones}

Se introduce el QR cuando se hace referencia a algún artículo, presentación o vídeo en la versión impresa de los escritos. Por ejemplo, en este artículo los QRs facilitan el acceso a vídeos relacionados con los puntos que se explican.

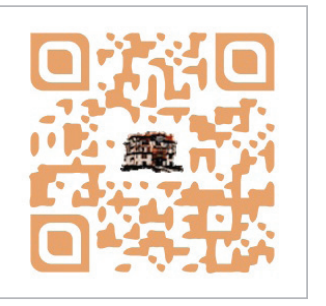

Figura 2. Biblioteca, ePub, QR y memoria local (vídeo de $\left.1^{\prime} 17^{\prime \prime}\right)^{5}$

\section{- Propaganda impresa}

En el verano de 2011 la campaña de promoción de la lectura de la biblioteca experimentaba con nuevas formas de llegar al usuario ${ }^{6}$. Se editó un folleto para ser buzoneado en el municipio y se creó un blog en el que se iban añadiendo las reseñas de los libros recomendados. El blog permitía seguir actualizando contenidos y, además, llevaba activada una opción para detectar el tamaño de las pantallas y adaptarse automáticamente a cualquier dispositivo. El folleto llevaba impreso el QR del blog.

EI QR, intermediario entre la información del papel y de la web, redirigía al blog de las novedades recomendadas, soslayando las limitaciones del papel (superficie impresa no actualizable) y proporcionando una experiencia de acceso satisfactoria a los dispositivos móviles.

\section{- Añadir contenidos personalizados al fondo}

Se ha añadido contenido multimedia (vídeos con entrevistas a los autores, reseñas) a libros del fondo de la biblioteca utilizando códigos QR. Es un proceso muy sencillo (tal y como se observa en el vídeo al que da acceso la figura 3) que puede ayudar a difundir en mejores condiciones la colección.

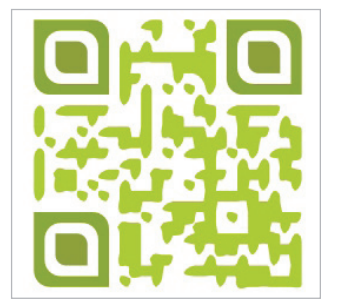

Figura 3. Vídeo "Bricolage tecnológico: libros y QR" (3'53") ${ }^{7}$

\section{Consejos a tener en cuenta: leer, crear, comunicar con QR}

\section{- Lectura de códigos}

Para acceder al contenido del QR necesitamos un dispositivo (teléfono/tablet) con cámara, una aplicación lectora de códigos QR y conexión a internet. Todos los smartphones del mercado tienen aplicaciones lectoras de QR (suelen ser aplicaciones gratuitas que vienen instaladas por defecto).

El proceso de lectura es muy simple: una vez localizado el código, abrimos la aplicación y enfocamos con la cámara; automáticamente se desencadena el proceso que nos lleva a la información almacenada (apertura de texto, tarjeta de visita, envío de sms, llamada telefónica, acceso al url...).

\section{- Creación de QRs}

Crear un QR también es muy sencillo y gratuito. Una simple búsqueda: "generar QR" en internet, llevará a webs que facilitarán el proceso ${ }^{8}$. Todas ofrecen un menú en el que hay que elegir el tipo de información que se va a encriptar (url, sms, texto, número de teléfono...) y decidir qué tamaño de $\mathrm{QR}$ se desea. Finalizado el proceso crea un archivo con la imagen.

Otra opción es usar el navegador. Tanto Chrome como Firefox permiten cargar extensiones para crear el QR del url activo. Una vez instalada, en Chrome aparece como opción del botón derecho del mouse, y en Firefox aparece un pequeño icono en la parte inferior derecha de la pantalla).

\section{EI QR sobre papel puede contener infor- mación fija o actualizable}

\section{- Estrategia de comunicación}

Una campaña de marketing QR consta de tres elementos básicos: el QR, el soporte donde imprimirlo y el contenido adaptado a la movilidad. Al poner a disposición de potenciales usuarios un QR se les está diciendo implícitamente varias cosas:

- es probable que tengas un dispositivo móvil con acceso a la Red;

- si es así, te mueves con ese dispositivo y esa movilidad te da libertad;

- desconocemos las condiciones en las que te encuentras cuando vayas a consumir lo que te proponemos, así que vamos a procurar que sea algo rápido y sencillo;

- somos conscientes de las limitaciones del tamaño de la pantalla y por ello vamos a procurar que aquello a lo que accedas se amolde a tu dispositivo.

Hay variables que no dependen de nosotros (tipo de dispositivo, condiciones de acceso) y otras que sí (tamaño del código, formato y calidad de la información suministrada). Un buen trabajo de implementación de QRs en nuestro entorno incidirá en un buen uso de las variables que controlamos para minimizar los riesgos de las que se nos escapan.

\section{Activador de información virtual en el entorno real}

Realidad aumentada ${ }^{9}$ (RA) es la superposición de información virtual (por ej., datos numéricos) a una imagen real (por ej., vista a través de una cámara). La información virtual enriquece la percepción sobre el entorno físico circundante.

La implantación de la realidad aumentada y su creciente popularización, superando el entorno del laboratorio al que parecía restringido, está íntimamente relacionada con la evolución del ecosistema de los dispositivos móviles (auge de smartphones y consolas, proliferación de aplicaciones que explotan los servicios basados en geolocalización, competencia entre los fabricantes por ofrecer dispositivos más 
potentes) y gran cantidad de objetos digitales existentes, creados por empresas y por ciudadanos.

Aunque todavía faltan unos años para su uso generalizado se intuye una forma de acceso mucho más sencilla de lo que se había previsto inicialmente. Los requerimientos técnicos se han simplificado, disponemos de mejores dispositivos (con mejor hardware, calidad de vídeo aceptable en las cámaras de nuestros teléfonos, capacidad de procesamiento suficiente), y las aplicaciones que dan soporte a esta tecnología se distribuyen a través de las plataformas más importantes facilitando su popularización. En el Informe APEI sobre web móvil se encuentra una descripción pormenorizada de esas aplicaciones (Arroyo-Vázquez, 2011).

\section{Elementos básicos}

Un servicio de realidad aumentada necesita 4 elementos:

1. Una cámara que capte las imágenes de la realidad que están viendo los usuarios, por ejemplo, las de los ordenadores o de los dispositivos móviles.

2. Un elemento sobre el que proyectar la mezcla de las imágenes reales con las imágenes sintetizadas. Se puede utilizar la pantalla de un ordenador, de un teléfono móvil o de una consola de videojuegos.

3. Hardware de procesamiento para interpretar la información del mundo real que recibe el usuario, generar la información virtual que cada servicio concreto necesite y mezclarla de forma adecuada. Nuevamente encontramos en los PCs, móviles y consolas estos elementos.

4. Un detonante que active el proceso de realidad aumentada. Puede ser una imagen que visualizan los usuarios, coordenadas de posición, etiquetas o marcadores del tipo RFID, códigos bidimensionales, o en general cualquier elemento que sea capaz de suministrar una información equivalente.

La computación en la nube y los dispositivos móviles han puesto de relieve el potencial multimedia del soporte papel

Analizando con detenimiento los 4 componentes no es dificil adivinar el por qué de la idoneidad de los teléfonos móviles para usos de RA (por defecto implementan los cuatro elementos con mayor o menor fortuna) y también es fácil entender que el papel, al permitir fijar en el mismo imágenes singulares, es un soporte apropiado para actuar como detonante.

\section{Realidad aumentada y bibliotecas. La experiencia de la Biblioteca de Muskiz}

Los escenarios de realidad aumentada aún son muy primarios (tanto en usabilidad como en funcionalidad) y la mezcla de realidad y virtualidad de forma cómoda y transparente todavía parece lejana. Depositar en este momento unas expectativas muy elevadas en el uso de esta tecnología puede producir un efecto de rechazo o minusvaloración. Sin embargo su potencial parece innegable, sobre todo en educación (Johnson; Adams; Cummins, 2012).

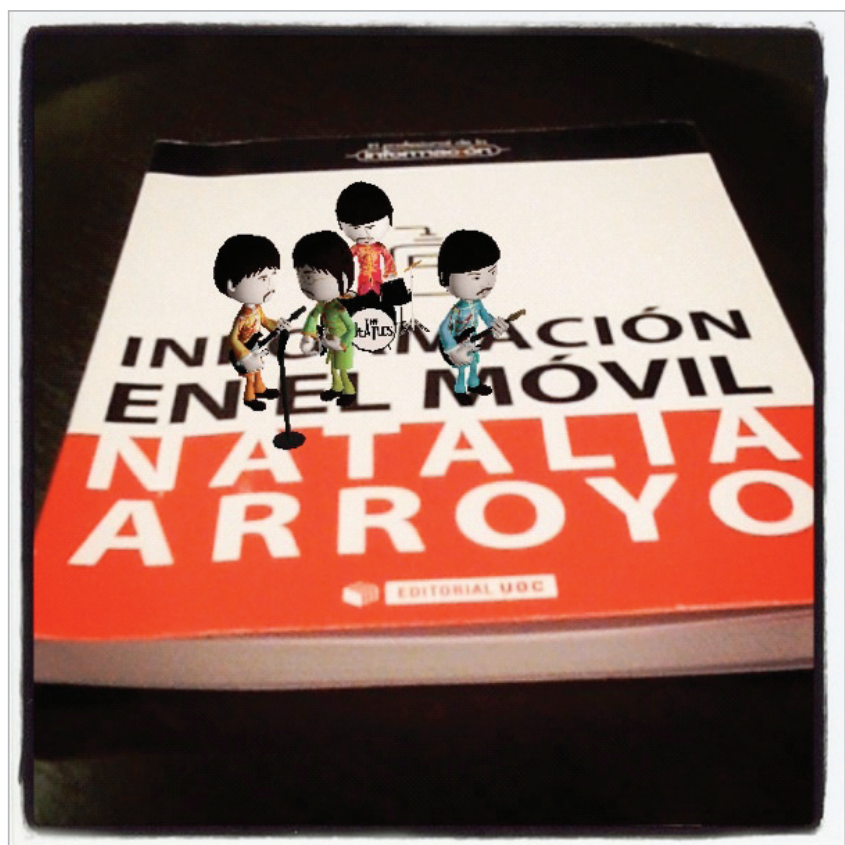

Figura 4. Ejemplo de RA basada en un "marcador-imagen". Al interactuar en un determinado contexto (usando la aplicación correspondiente, en condiciones óptimas de acceso a la web, con un dispositivo con potencia suficiente...) la cubierta del libro es el "detonante" del proceso de realidad aumentada

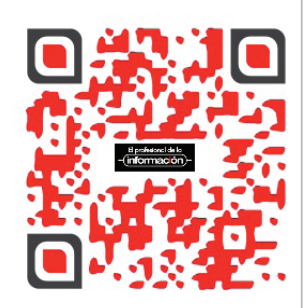

Figura 5. QR de acceso al vídeo que muestra el proceso en funcionamiento $\left(53^{\prime \prime}\right)^{10}$

En las bibliotecas también se está empezando a experimentar con el uso de estas aplicaciones (Hahn, 2012). La RA facilita una toma de contacto didáctica e informal con los dispositivos móviles y ofrece muchas oportunidades de marketing. La mayoría de las experiencias piloto realizadas se han centrado en el geoposicionamiento (creación de capas para localización de centros ${ }^{11}$ y la difusión del patrimonio histórico ${ }^{12}$ ).

El primer uso de realidad aumentada en Muskiz también empleaba el geoposicionamiento, en este caso para difundir el patrimonio inmaterial local (Riancho; Juárez-Urquijo, $2011)^{13}$ pero en este artículo se pretende resaltar las posibilidades de los marcadores-imagen, con el papel como soporte, para crear nuevos servicios bibliotecarios. El soporte impreso, tan abundante en nuestras bibliotecas (libros de novedades, folletos de propaganda), es el que sirve de engarce entre la biblioteca tradicional y sus nuevos servicios multimedia. Los siguientes ejemplos ilustran las posibilidades del soporte papel para experimentar con la realidad aumentada.

\section{- Recomendaciones de lectura ${ }^{14}$}

Son vitales en el día a día de nuestros centros, y los usuarios suelen intercambiarlas entre ellos a pie de mostrador. Se grabó a una lectora explicando por qué le había gustado un libro y animando a su lectura ${ }^{15}$. Después se empleó una 
aplicación de realidad aumentada -Aurasma ${ }^{16}$ - que permite asociar el vídeo con una imagen (la propia cubierta del libro que se quiere recomendar). En la sección de novedades, al enfocar el libro con un dispositivo móvil que tenga instalada la aplicación, el vídeo aparece superpuesto en la cubierta.

Pareció interesante esta actividad porque además de aprender a utilizar la tecnología -todo el proceso se realizó con un smartphone-, se difundía de forma lúdica su utilización implicando a los usuarios. Este marketing enlaza con la tradición de la biblioteca (las recomendaciones de lectura) y le otorga un "plus" de institución que evoluciona.

Aurasma es fácil de usar para el programador, pero penaliza en exceso la experiencia al usuario final que accede con su propio dispositivo, obliga a darse de alta para acceder a los contenidos generados por terceros, la captación no es intuitiva y exige esfuerzo y paciencia. Sin embargo es recomendable para entornos en los que todo el proceso de RA esté bajo control (por ejemplo museos o bibliotecas con sala de exposiciones que ofrezcan los dispositivos a modo de guías interactivas).

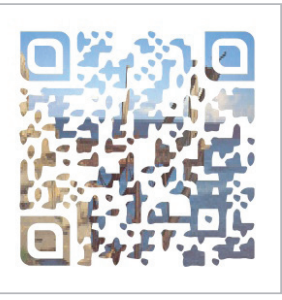

Figura 6. QR de acceso al vídeo "Recomendaciones de lectura y realidad aumentada" $\left(43^{\prime \prime}\right)^{17}$

\section{- Realidad aumentada en las publicaciones impresas ${ }^{18}$}

Layar $^{19}$, una de las aplicaciones estrella de RA basada en geoposicionamiento, posibilita con Creator $^{20}$ integrar botones interactivos en el material impreso. A diferencia de Aurasma, con Layar Creator podemos asignar funciones personalizadas a los elementos virtuales que incrustemos al marcador-imagen (abrir una página web, realizar una llamada telefónica, tuitear, ver un vídeo...) dotando de interactividad al impreso, que se convierte así en un producto multimedia.

\section{Consejos a tener en cuenta}

Las bibliotecas son centros que reúnen condiciones óptimas para experimentar con esta tecnología: ofrecen buena cobertura wifi, poseen objetos singulares sobre los que agregar elementos virtuales (que además son buenos activadores de aplicaciones de realidad aumentada), disponen de dispositivos para que los usuarios puedan familiarizarse con los nuevos servicios, personal cualificado, y pueden usar aplicaciones gratuitas que no penalizan los recursos económicos.

Ya hemos comentado que hay aplicaciones de realidad aumentada que utilizan el geoposicionamiento como activador (por ejemplo las capas de Layar para localizar bibliotecas) y otros que usan marcadores imagen; en ambos casos las características del dispositivo determinarán la calidad del servicio.

De la misma manera que no sabemos qué tipo de dispositivo utilizará el usuario para leer en pantalla (y por eso es
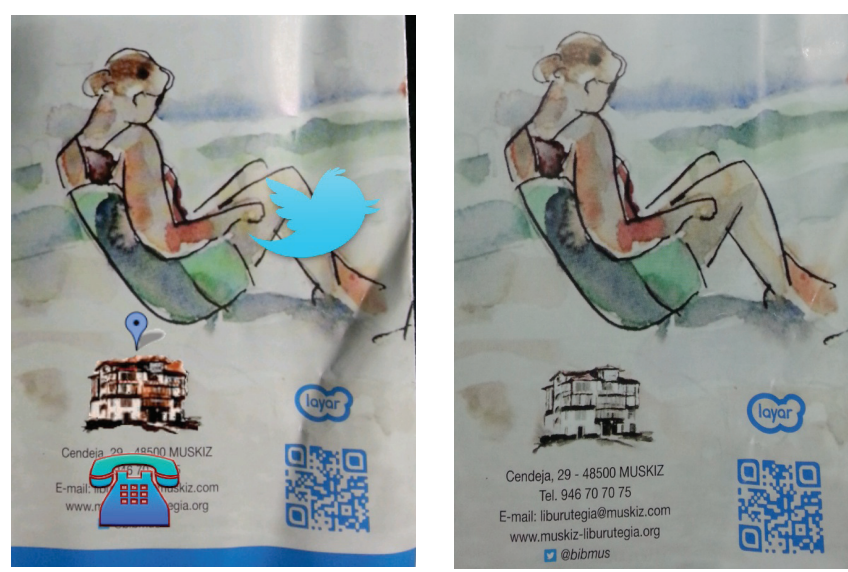

Figuras 7 y 8 . Imagen del folleto con y sin RA

conveniente hacer una apuesta por los formatos abiertos) o para acceder a los QRs, desconocemos también qué dispositivo empleará para los servicios de RA. Es aconsejable que el servicio sea compatible para las dos grandes plataformas actuales (Android e IOS) y que los requerimientos técnicos sean lo más sencillos posibles, evitando imágenes o vídeos muy pesados, que exigen una gran capacidad de procesamiento, y un ancho de banda del que tal vez no se disponga. Como siempre, para una biblioteca es interesante utilizar tecnologías multiplataforma.

\section{Conclusiones}

La aparición y el despliegue de una nueva tecnología conlleva a veces nuevas oportunidades de evolución para herramientas y aplicaciones que en un principio parecían haberse quedado obsoletas. La computación en la nube y los dispositivos móviles han puesto de relieve el potencial multimedia del soporte papel.

La evolución de las tecnologías de la información y la aparición de nuevos soportes y formas de difusión hacen necesario un replanteamiento de los servicios tradicionales de la biblioteca

La evolución de las tecnologías de la información y la aparición de nuevos soportes y formas de difundir las ideas hacen necesario un replanteamiento de los servicios tradicionales de la biblioteca. Adoptando un espíritu abierto, innovador y creativo, las bibliotecas pueden acometer proyectos tecnológicos que renueven los servicios bibliotecarios y beneficien a sus usuarios.

Los dispositivos móviles ayudan a romper las barreras físicas de la biblioteca y están cambiando los hábitos y la forma de llegar a la información y, por lo tanto, también la forma de dispensar los servicios, cada vez más accesibles desde cualquier lugar.

El acceso a la información desde dichos equipos se encuentra en un estadio incipiente y no está aún consolidado. Este escenario inicial es propicio para la realización de pruebas 
que ayuden a comprender mejor el nuevo entorno. Podemos empezar por soluciones sencillas, empleando herramientas gratuitas para experimentar y probar, como paso previo a futuros servicios más avanzados y costosos.

Una de las mayores dificultades es la falta de equipos propiedad de la biblioteca. En este sentido es necesario incidir en la necesidad de dotar a los centros con esas herramientas para poder seguir ofreciendo servicios adaptados a las nuevas necesidades.

\section{Notas}

1. Soporte resistente al paso del tiempo que facilita el acceso a la información que contiene sin necesidad de dispositivos.

2. Un ejemplo es intentar introducir el url de algún recurso citado en un artículo de revista en una tablet o en un teléfono. Hay que estar muy interesado para hacerlo.

3. Lo que en la práctica supone codificar información más detallada.

4. http://www.muskiz-liburutegia.org

5. http://youtu.be/3aRpqPtM90o

6. Juárez-Urquijo, Fernando (2011). “Marketing BB”. Biblioblog.

http://diarium.usal.es/biblioblog/2011/2948

\section{7. http://youtu.be/nVFxqMaPoSk}

8. Algunas de las más conocidas son: Kaywa, Zxing Project, GOQR.ME, Unitag.

9. http://es.wikipedia.org/wiki/Realidad_aumentada

\section{0. $h t t p: / / y o u t u . b e / E O d X J V C J k L 8$}

11. Mediante la creación de capas para localizar las bibliotecas más cercanas al punto en el que se encuentra el usuario. En España la Subdirección General de Coordinación Bibliotecaria del Ministerio de Cultura ha creado una capa en Layar a través de la cual se pueden localizar más de 8.200 bibliotecas de España y otros países

http://m.layar.com/open/bibliotecas2011 (se recomienda acceder desde un smartphone IOS o Android).

12. Con capas que posicionan las fotos de los archivos históricos en su emplazamiento original (mostrando monumentos, arquitectura, paisajes), como la realizada en Philadelphia.

http://www.azavea.com/research/company-research/ augmented-reality

13. Una app para entender la Batalla de Somorrostro de 1874.

http://muskizliburutegia.blogspot.com.es/2012/02/unaapp-para-entender-la-batalla-de.html

14. Juárez-Urquijo, Fernando (2011). "Recomendaciones de lectura y realidad aumentada". Biblioblog. http://diarium.usal.es/biblioblog/2011/recomendacionesde-lectura-y-realidad-aumentada

15. El vídeo debe ser breve, por razones de peso y para no cansar al que lo vaya a ver.

16. http://www.aurasma.com

17. http://youtu.be/vgPbbgWBQXY

18. http://muskizliburutegia.wordpress.com/2012/07/24/ un-folleto-muy-especial-realidad-aumentada-con-layar

19. $h t t p: / / w w w . l a y a r . c o m$

20. http://www.layar.com/blog/2012/06/05/introducinglayar-creator

\section{Bibliografía}

Abarca-Villoldo, Marta; Pons-Chaigneau, David-Marcelo; Rubio-Montero, Francisco-José; Vallés-Navarro, Raquel (2011). "QR codes in use: the experience at the UPV Library". Serials, v. 24, n. 3, suplement, pp. S47-S56.

http://hdl.handle.net/10251/14112

http://dx.doi.org/10.1629/24S47

Arroyo-Vázquez, Natalia (2009). "Web móvil y bibliotecas". El profesional de la información, v. 18, n. 2, pp. 129-136. http://hdl.handle.net/10760/13057 http://dx.doi.org/10.3145/epi.2009.mar.02

Arroyo-Vázquez, Natalia (2011). Informe APEI sobre movilidad. Gijón: Asociación Profesional de Especialistas en Información. ISBN 9788469456552.

http://eprints.rclis.org/15898/1/informeapeimovilidad.pdf

Hahn, Jim (2012). "Mobile augmented reality applications for library services". New library world, v. 113, n. 9/10, pp. 429-438.

http://dx.doi.org/10.1108/03074801211273902

Johnson, Larry; Adams, Samantha; Cummins, Michele (2012). The NMC Horizon report: 2012 higher education edition.

http://net.educause.edu/ir/library/pdf/HR2012.pdf

Leiva-Aguilera, Javier (2012). "Introducción a los códigos QR y algunos usos". Anuario ThinkEPI, v. 6, pp. 309-312.

Riancho, Gontzal; Juárez-Urquijo, Fernando (2011). “Muskiz y Las batallas de Somorrostro: un ejemplo práctico de cómo entendemos la memoria local en la sociedad de la movilidad". En: IV Encuentro bibliotecas y municipio, Madrid, 17 nov.

http://travesia.mcu.es/portalnb/jspui/bitstream/10421/6146/1/ Muskiz.pdf

Walsh, Andrew (2010). "QR codes: using mobile phones to deliver library instruction and help at the point of need". Journal of information literacy, v. 4, n. 1, pp. 55-65.

http://ojs./boro.ac.uk/ojs/index.php/JIL/article/view/ LLC-V4-I1-2010-1/1450 